\title{
Sec62 promotes early recurrence of hepatocellular carcinoma through activating integrina/CAV1 signalling
}

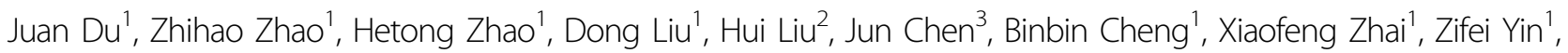 \\ Yani Zhang ${ }^{1}$ and Changquan Ling ${ }^{1}$
}

\begin{abstract}
Postsurgical recurrence within 2 years is the major cause of poor survival of hepatocellular carcinoma (HCC) patients. However, the molecular mechanism underlying $\mathrm{HCC}$ recurrence remains unclear. Here, we distinguish the function and mechanism of Sec62 in promoting HCC recurrence. The correlation between Sec62 and early recurrence was demonstrated in $60 \mathrm{HCC}$ samples from a prospective study. HCC cells with Sec62 knockdown $\left(S e c 62^{\mathrm{KD}}\right.$ ) or overexpression $\left(\mathrm{Sec} 62^{\mathrm{OE}}\right.$ ) were used to determine the potential of Sec62 in cell migration in vitro. Microarray analysis comparing Sec62 $2^{K D}$ or Sec62 ${ }^{O E}$ to their control counterparts was used to explore the mechanisms of Sec62-induced recurrence. A luciferase-labelled orthotopic nude mouse model of HCC with Sec62 ${ }^{K D}$ or Sec62 ${ }^{O E}$ was used to validate the potential of Sec62 in early HCC recurrence in vivo. We found that high expression of Sec62 was positively correlated with surgical recurrence in clinical HCC samples. Multivariate analysis revealed that Sec62 was an independent prognostic factor for early recurrence in postoperative HCC patients. Moreover, Sec62 promoted migration and invasion of HCC cells in vitro and postsurgical recurrence in vivo. Mechanically, integrina/ CAV1 signalling was identified as one of the targets of Sec62 in cell movement. Overexpression of integrin a partially rescued the Sec62 knockdown-induced inhibition of cell migration. Sec62 is a potentially prognostic factor for early recurrence in postoperative HCC patients and promotes HCC metastasis through integrina/CAV1 signalling. Sec62 might be an attractive drug target for combating HCC postsurgical recurrence.
\end{abstract}

\section{Introduction}

Hepatocellular carcinoma (HCC) was the fourth leading cause of cancer death worldwide in 2018, with $\sim 841,000$ new cases and 782,000 deaths annually ${ }^{1}$. The major risk factors of $\mathrm{HCC}$ vary from region to region. In highest incidence areas of HCC (China, Eastern Africa), the key determinants are chronic hepatitis B virus (HBV) infection and aflatoxin exposure, whereas in other countries (Japan, Egypt), HCV infection is likely the predominant

\footnotetext{
Correspondence: Changquan Ling (changquanling@smmu.edu.cn)

${ }^{1}$ Department of Traditional Chinese Medicine, Changhai Hospital, Navy Military

Medical University, Shanghai 200433, China

${ }^{2}$ The Third Department of Hepatic Surgery, Eastern Hepatobiliary Surgery

Hospital, Navy Military Medical University, Shanghai 200433, China

Full list of author information is available at the end of the article.

These authors contributed equally: Juan Du, Zhihao Zhao
}

cause $^{2}$. Notably, the mortality rates of HCC have increased in northern and central Europe and the USA, where the leading risk factors include alcohol consumption, increased overweight/obesity, and diabetes ${ }^{3}$. Surgical resection, liver transplantation or local ablation are recommended for patients with very early stage tumours (Barcelona Clinic Liver Cancer [BCLC] 0/A). Liver transplantation is difficult to perform due to its high cost and the scarcity of donor organs in Asian-Pacific countries ${ }^{4}$. For patients with well-preserved hepatic function, surgical resection provides a better clinical outcome than local ablation ${ }^{5,6}$. However, tumour recurrence commonly occurs within two years of resection (19\% to $25 \%$ in the first year) and exceeds $70 \%$ at 5 years ${ }^{7,8}$. Metastasis is the major reason for the high recurrence and poor survival of

\section{(c) The Author(s) 2019}

\footnotetext{
(c) Open Access This article is licensed under a Creative Commons Attribution 4.0 International License, which permits use, sharing, adaptation, distribution and reproduction cc) in any medium or format, as long as you give appropriate credit to the original author(s) and the source, provide a link to the Creative Commons license, and indicate if changes were made. The images or other third party material in this article are included in the article's Creative Commons license, unless indicated otherwise in a credit line to the material. If material is not included in the article's Creative Commons license and your intended use is not permitted by statutory regulation or exceeds the permitted use, you will need to obtain permission directly from the copyright holder. To view a copy of this license, visit http://creativecommons.org/licenses/by/4.0/.
} 
HCC patients ${ }^{9,10}$. Therefore, it is important to elucidate the molecular mechanisms of HCC metastasis.

Mammalian Sec62 is associated with the Sec61-complex, the main pore for protein translocation in the ER membrane, and with Sec63, an ER membrane protein containing a luminal J-domain ${ }^{11}$. Mammalian Sec62 also interacts with ribosomes via two conserved peptide motifs in its $\mathrm{N}$-terminal cytosolic domain ${ }^{12,13}$. Through these functional elements, Sec62 regulates translation, influences protein translocation to the ER, and indirectly interacts with the key regulator of the unfolded protein response (UPR), BiP (Grp78, HspA5), thereby affecting cellular responses to ER stress inducers. Sec62 was previously characterised as a probable target gene in prostate cancer, lung cancer and thyroid carcinoma due to its high positive rate. By silencing SEC62, cell migration and the invasive potential of prostate cancer cells are markedly reduced, while cell viability is minimally affected ${ }^{14}$. To date, no studies have reported the clinicopathologic significance and potential function of Sec62 in HCC.

Integrins are a group of heterodimeric adhesion receptors that mediate the attachment of cells to extracellular matrices and other cells. Some aspects of integrin function are dependent on the interactions of integrin with its neighbours in the cell membrane and inside the cell. Caveolin, which is a src kinase substrate, is a 210 amino acid $(\sim 21 \mathrm{kDa})$ membrane protein that binds cholesterol and a number of signalling molecules potentially linked with integrin function. Integrin plays a role in migration and tumour metastasis of tumour cells ${ }^{15}$. The integrin $\alpha \mathrm{V}$, 5 and 11 subunits have been reported to be involved in the process of HCC cell migration ${ }^{16-20}$. Moreover, targets of integrins, MLCK and calpain, are required for the invasive and metastatic potential of tumour cells ${ }^{21-23}$.

In this study, we demonstrate that high expression of Sec62 is associated with surgical recurrence of HCC. Sec62 promotes HCC cell migration and invasion by upregulating integrin $\alpha / C A V 1$ expression. Sec62 might be a prognostic marker and an attractive drug target for combating the postsurgical recurrence of HCC.

\section{Results}

High expression of Sec62 promotes early recurrence of $\mathrm{HCC}$ after curative resection in patients

The function and clinical relevance of Sec62 have been poorly described in human HCC. In this study, we evaluated the immunohistochemical expression of Sec62 in tumour tissues from a prospective cohort of $60 \mathrm{HCC}$ patients. After 2 years of follow-up, the levels of Sec62 were much higher in tumour tissues with early recurrence $(20 / 60)$ than in non-recurrent tumours (40/60) (Fig. 1a left and Fig. 1Sa). We further analysed the correlation between Sec62 expression and early HCC recurrence. The expression levels of Sec62 were positively correlated with early HCC recurrence (Fig. 1a right). Meanwhile, Western blot analysis showed that the Sec62 levels in tumour

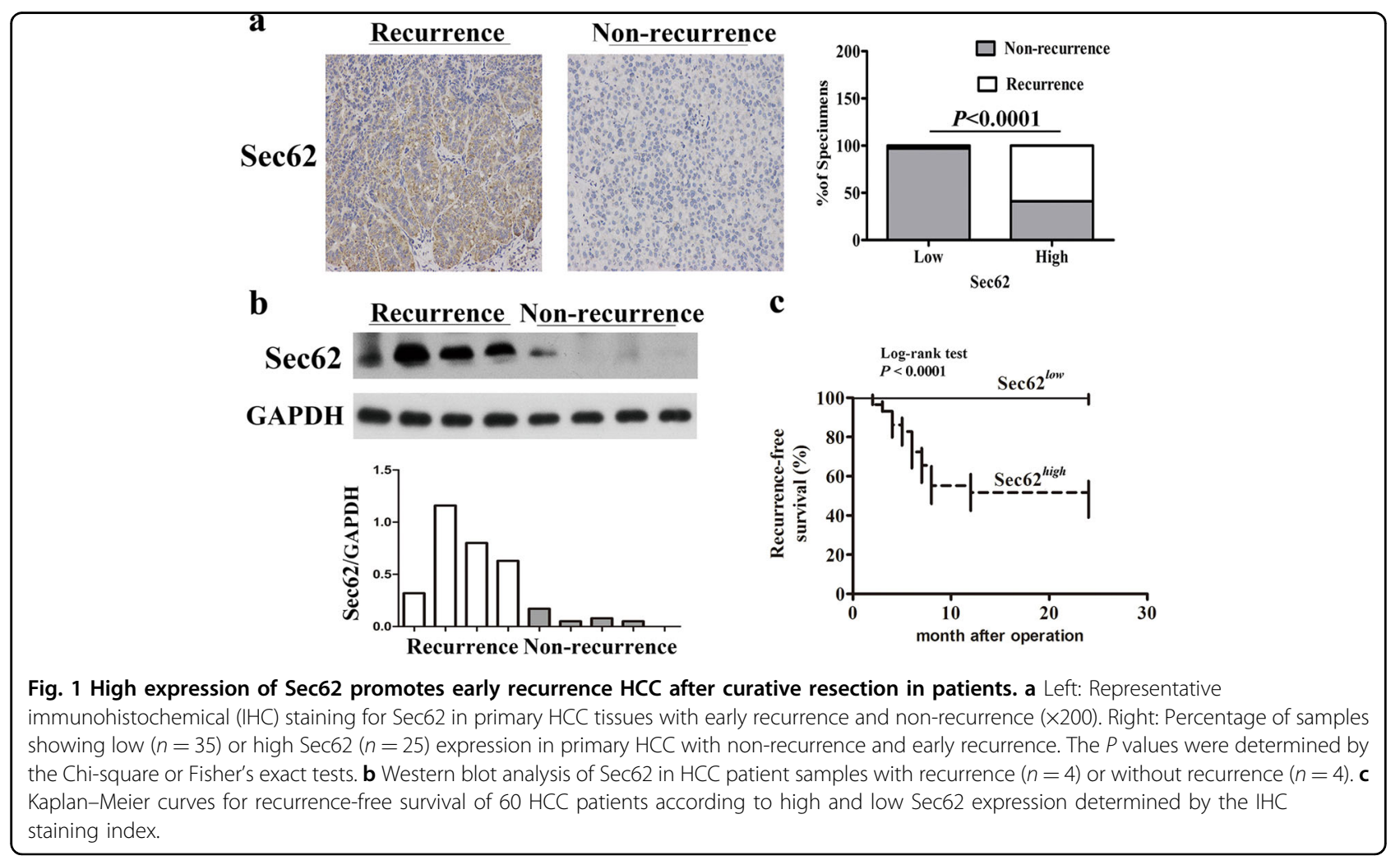




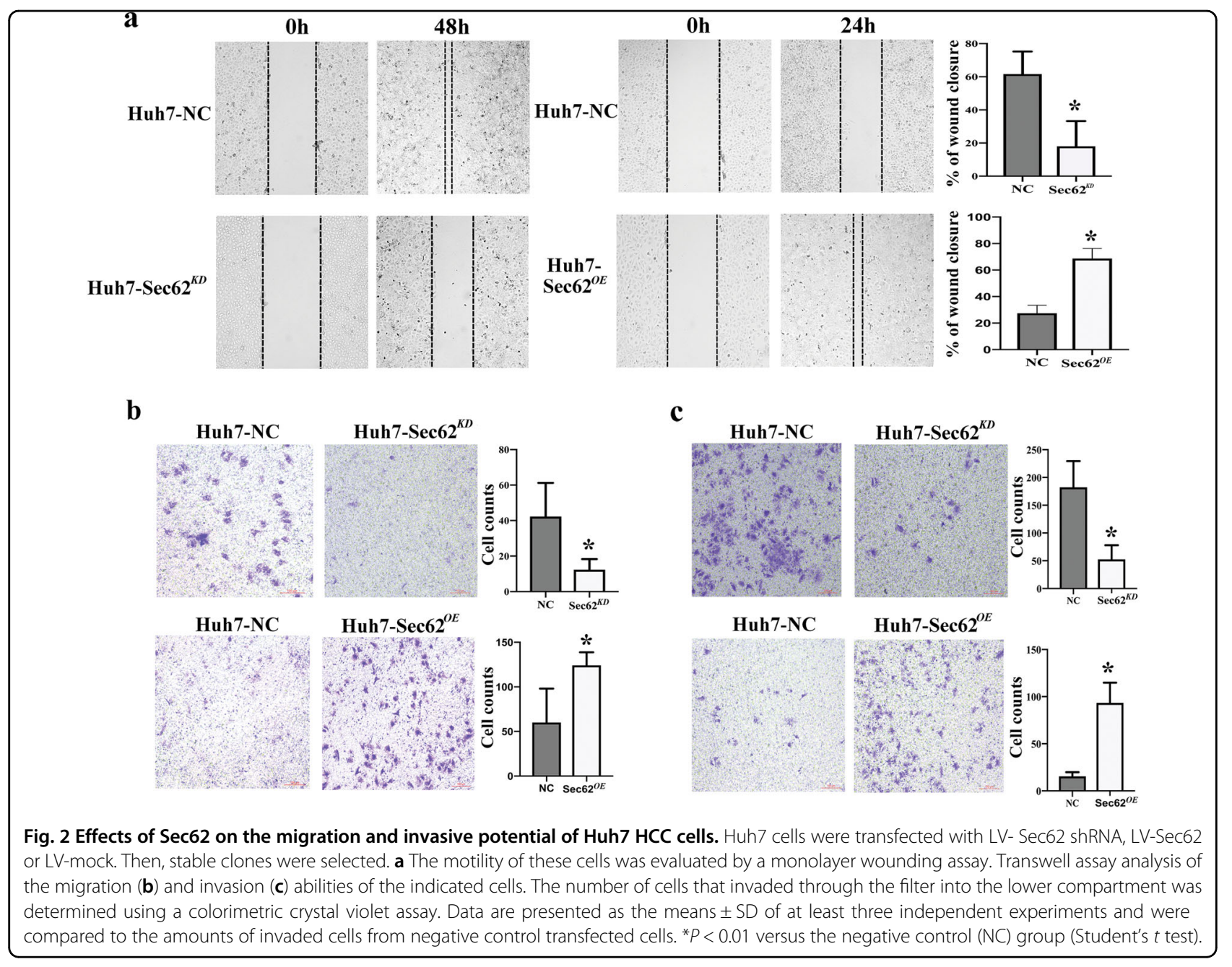

tissues with early recurrence were much higher than those from non-recurrent tumours (Fig. 1b). Furthermore, higher expression of Sec62 was associated with shorter recurrence-free survival of patients. Multivariate analysis revealed that Sec62 expression was an independent and significant risk factor for HCC recurrence (Fig. 1c). The area under the ROC curve (AUC) was 0.89 (95\% CI $0.81-0.98$ ) (Fig. 1Sb). These results suggest that Sec62 is closely correlated with early recurrence and may be a potential predictor for postsurgical recurrence of HCC.

\section{Sec62 promotes HCC cell migration and increases its invasive potential}

The protein and mRNA levels of Sec62 in Huh7, Hep G2 and 97H cells were higher than those in the Hep3B, MHCC97-L, and LM3 cell lines as well as human normal liver cells, L02 (Fig. 2Sa). To determine the role of Sec62 in HCC cells in vitro, we first determined whether Sec62 had any effect on HCC cell proliferation. Two stable cell lines, Huh7-Sec62 ${ }^{K D}$ and Huh7-Sec62 ${ }^{O E}$, were established. The MTT assay showed that down-regulation or up-regulation of Sec62 had no effect on Huh7 cell proliferation at $24 \mathrm{~h}$ (Fig. $2 \mathrm{Sb}$ ).

Cell migration and invasion are characteristics of most malignant tumours. Next, we determined the impact of Sec62 on cell migration and invasion. As shown in Fig. 2a-c, down-regulation of Sec62 decreased the migration and invasion of Huh7 cells, whereas up-regulation of Sec62 expression significantly increased the migration and invasion of Huh7 cells. Using 97H and 97L cells, similar results were observed, as shown in Fig. 3Sa-c: down-regulation of Sec62 decreased the migration and invasion of $97 \mathrm{H}$ cells. Up-regulation of Sec62 expression increased the migration and invasion of 97L cells. These results suggest that Sec62 may promote postsurgical recurrence of HCC by increasing HCC cell migration and invasion.

\section{Sec62 promotes cellular movement via targeting integrin a/CAV1 signalling}

To elucidate the molecular mechanisms by which Sec62 contributes to the migration and invasion of HCCs, we carried out microarray analyses comparing the gene 
expression of Huh7Lv-shSec62 versus Huh7Lv-NS cells and Huh7Lv-Sec62 versus Huh7Lv-NS cells. Gene expression profiling using the Affymetrix GeneChip PrimeView Human Gene Expression Array identified 331 up-regulated and 534 down-regulated transcripts and 146 up-regulated and 45 down-regulated transcripts, which were significantly differentially expressed based on the | $\mathrm{FC} \mid>1.5$ and $\mathrm{P}<0.01$ thresholds, in Huh7 cells with Sec62 knockdown and Sec62 overexpression, respectively, compared with their controls. Moreover, functional gene analysis using Ingenuity Pathway Analysis (IPA) revealed that Sec62 knockdown modulated key pathways typically activated in IL-6 signalling, PPAR signalling, integrin signalling, PI3K/AKT signalling and phospholipase C signalling, while Sec62 overexpression modulated key pathways typically activated in IL-8 signalling, integrin signalling and phospholipase $\mathrm{C}$ signalling ( $\mid \mathrm{Z}$-score $\mid>2$, Fig. 3a left). Notably, the integrin pathway and phospholipase $\mathrm{C}$ pathway were the common putative signalling pathways identified by Sec62 knockdown and overexpression. Next, ten functional classifications, as annotated by Gene Ontology (GO), were significantly enriched, including cell movement, cellular growth and proliferation, cancer, etc. Cell movement was the most modulated function following both Sec62 knockdown and overexpression (Fig. 3a upper right). Based on the $p$-value and the Gene Ontology analysis, integrin signalling was selected for further analysis. The knowledge-based interactome of Sec62-regulated integrin in Huh7-Sec62 knockdown and Huh7-Sec62 overexpression cells was constructed using IPA and is shown in Fig. 4Sa. To further identify the pathway regulated by Sec62, we examined the knowledge-based integrin pathway using IPA overlaid with microarray data from Huh7-Sec62 knockdown cells (Fig. 3a lower right). Several members of the integrin pathway regulated by Sec62 were confirmed by qRT-PCR and western blot analysis. Among them, the expression of integrin $\alpha 2 b$, integrin $\alpha 4$, integrin $\alpha 5$, integrin $\alpha \mathrm{V}, \mathrm{CAV} 1$, calpain, and MLCK are consistent with microarray data from both qRT-PCR and Western blot analysis (Fig. $4 \mathrm{Sb}, \mathrm{c}$ ).

To validate the role of integrin $\alpha$ in Sec62-induced HCC cell migration and invasion, we overexpressed integrin $\alpha 2 b$, integrin $\alpha 4$, integrin $\alpha 5$, and integrin $\alpha \mathrm{V}$ in Sec62knockdown Huh 7 cells and determined their effects on cell migration and invasion. As shown in Fig. 3b and 5S, down-regulation of Sec62 decreased the migration of Huh7 cells, whereas ectopic expression of integrin $\alpha 5$ and integrin $\alpha \mathrm{V}$ significantly abrogated Sec62 knockdown-induced inhibition of cell migration. Ectopic expression of integrin $\alpha 2 \beta$, $\alpha 4, \alpha 5$ and integrin $\alpha \mathrm{V}$ were validated by Western blotting (Fig. 3b and 5S). To further explore the targets of Sec62 that contribute to the corresponding changes in integrin $\alpha$ signalling, co-IP was carried out in Huh7 cells. The results demonstrated that integrin $\alpha \mathrm{V}$ or integrin $\alpha 5$ were immunoprecipitated and Sec62 was co-immunoprecipitated together with integrin $\alpha \mathrm{V}$ or integrin $\alpha 5$ (Fig. 3b right). Coincidentally, the levels of integrin $\alpha \mathrm{V}$ and integrin $\alpha 5$ as well as the integrin $\alpha$ targets CAV1, calpain, and MLCK expression in HCC samples with early recurrence were higher than those in non-recurrence samples (Fig. 3c). These results further indicate that Sec62-induced cell migration is dependent on integrin $\alpha \mathrm{V}$ and integrin $\alpha 5$.

\section{Sec62 is involved in postsurgical recurrence of HCC in the orthotopic xenograft mouse model of HCC}

To further investigate the function of Sec62 in early HCC recurrence, we generated mice bearing orthotopically implanted tumours formed from luciferase-labelled $\mathrm{Lv}-\mathrm{Sec} 62^{K D}, \mathrm{Lv}-\mathrm{Sec} 62^{O E}$, or Lv-NC Huh7 cells. Within 14 days after surgical resection, five out of seven mice bearing Lv-NC cells had relapsed with metastasis. In marked contrast, no recurrence was observed in mice bearing Lv-Sec62 ${ }^{K D}$ cells (Fig. $4 \mathrm{a}$ and b left). While $100 \%$ of mice bearing Lv-Sec62 ${ }^{O E}$ cells relapsed within 10 days after surgical resection, but only a fraction of mice $(2 / 7)$ bearing Lv-NC cells relapsed (Fig. 4a and b right). Western blot analysis showed that Sec62 expression and Sec62 targets integrin $\alpha / \mathrm{CAV} 1$ expression in tumour tissues from the surgical resection in the Sec $62^{K D}$ group were much lower than that in the $\mathrm{NC}$ group (Fig. 4c left), while Sec62 expression and integrin $\alpha /$ CAV1 expression in the Sec62 ${ }^{O E}$ group was much higher than that in the NC group (Fig. 4c right). Collectively, these results suggest that high expression of Sec62 promotes postsurgical recurrence of $\mathrm{HCC}$ in an orthotopic xenograft mouse model.

\section{Discussion}

$\mathrm{HCC}$ recurrence is a major postoperative complication. In particular, early recurrence (i.e., within 2 years of resection) accounts for more than $70 \%$ of tumour recurrence ${ }^{24}$. Identification of patients who are at high risk of recurrence after potentially curative surgery allows clinicians to detect recurrent $\mathrm{HCC}$ at its earliest stage, when curative therapy may still be feasible. Potential biomarkers for identification and the mechanisms underlying rapid recurrence need to be explored. Here, we demonstrate the strong promotion potential of Sec62 for HCC early recurrence through activating integrin $\alpha /$ CAV1 signalling. Sec62 might be a prognostic biomarker and an attractive therapeutic target for combating postoperative $\mathrm{HCC}$ recurrence.

In recent years, numerous studies have reported the Sec62's relevance, as a prognostic biomarker to head and neck cancer ${ }^{25,26}$, prostate cancer ${ }^{27}$, and lung cancer ${ }^{22,25}$, indicating a worse outcome for patients with higher Sec62 levels. On the contrary, another cohort of prostate cancer patients with an increased SEC62 level showed a lower 


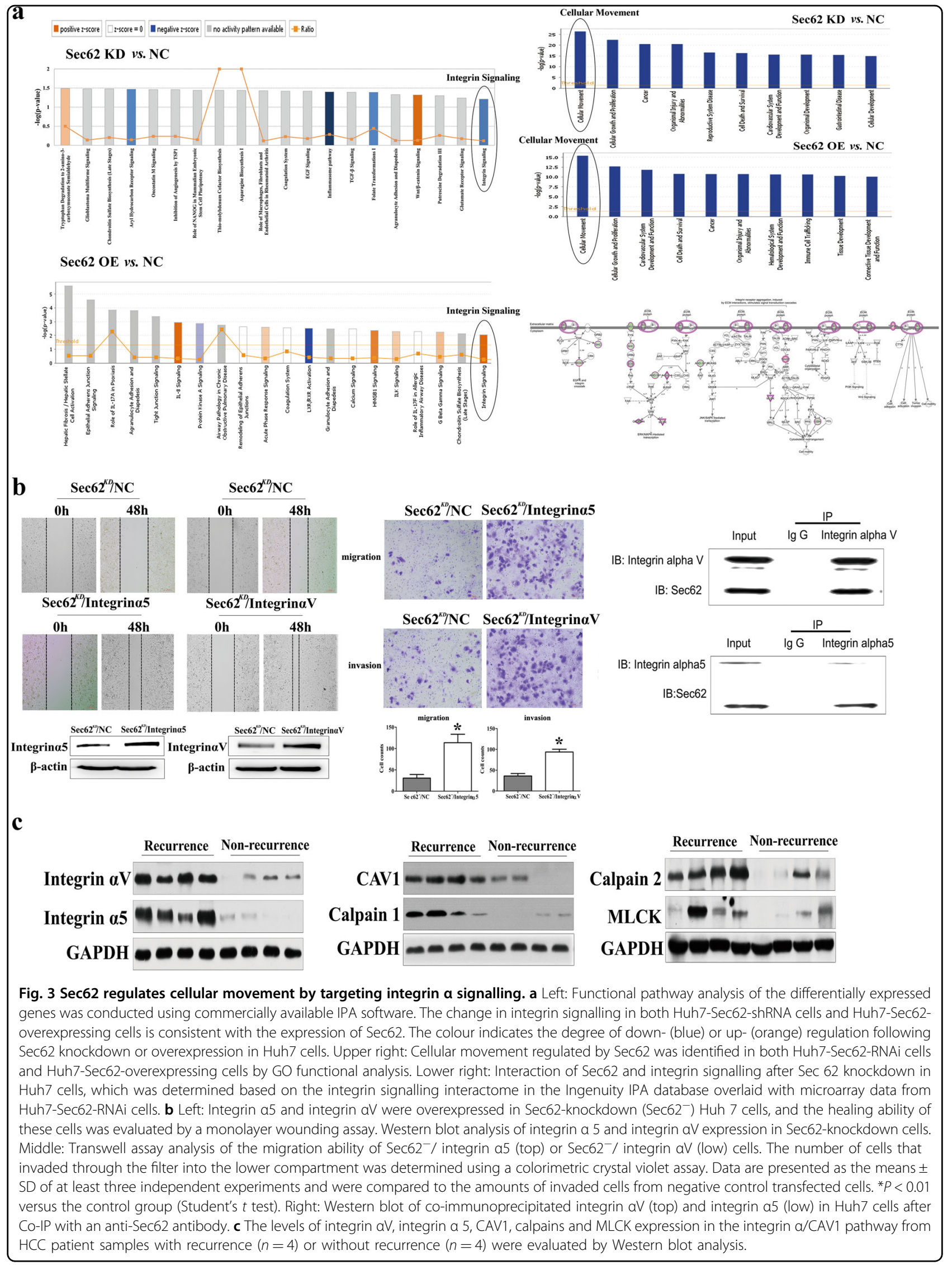




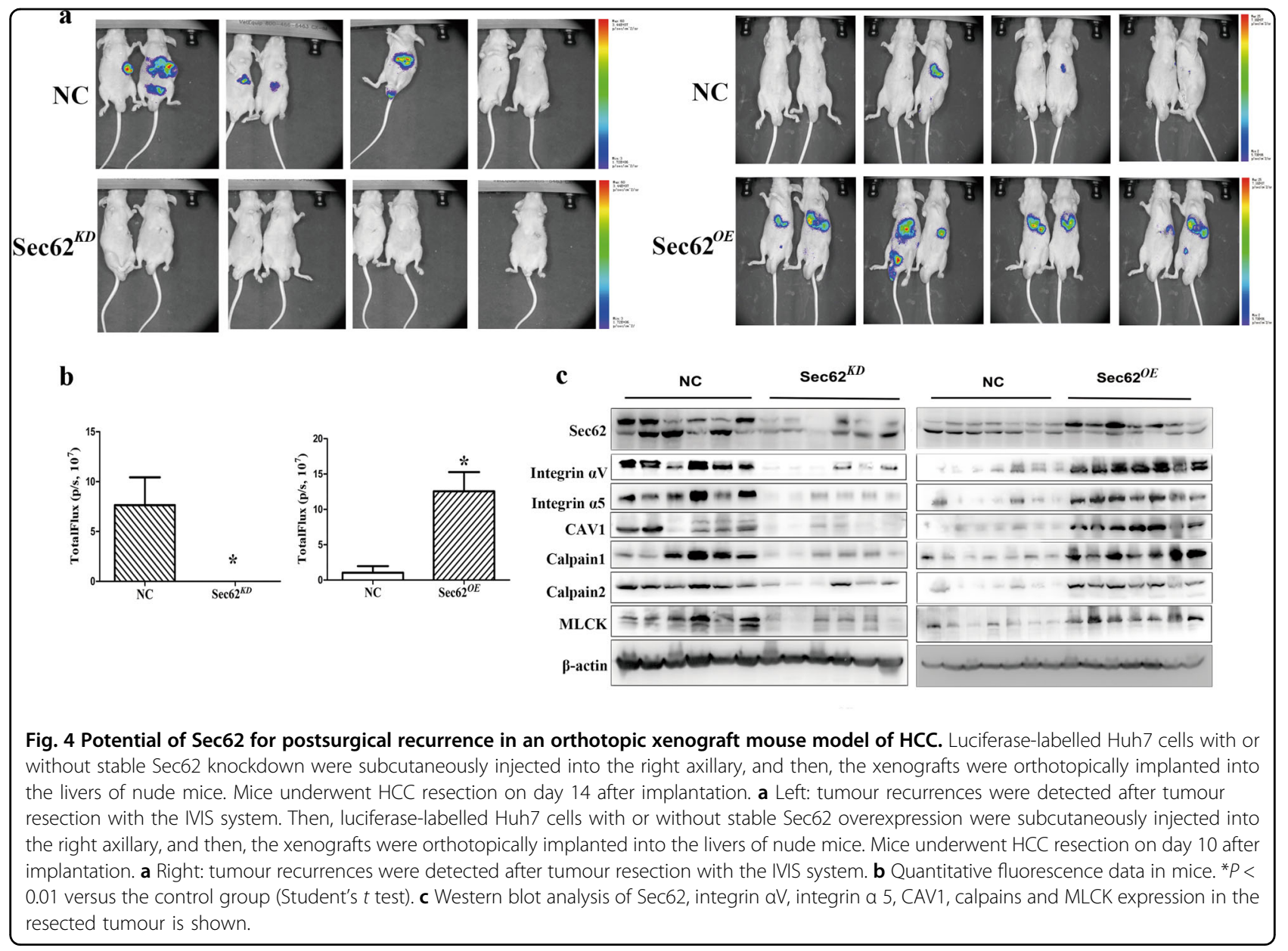

risk of recurrence and progression compared with patients without an increased SEC62 level, which might be due to the comparably few patients in this study $(n=22)^{28}$. In the present prospective study, we found that HCC patients (BCLC A) with high expression of Sec62 had higher recurrence rates than patients with low expression of Sec62 within 2 years after surgical resection. By multivariate analysis, Sec62 was an independent and significant risk factor for HCC recurrence. These clinical data are consistent with previous research and strongly suggest that Sec62 plays a role in the early surgical relapse of HCC. To further validate the potential role of Sec62 as a prognostic marker and to establish a valid basis for clinical applications, tumours from more surgical HCC patients need to be studied in the future.

According to our in vitro study, the levels of Sec62 in Huh7, Hep G2 and 97H cells were higher than those in other cell lines. To explore the roles of Sec62 in HCC cell biology, Sec62 knockdown and overexpression in Huh7, 97H and 97L cells were investigated. Our results clearly showed that knockdown or overexpression of Sec62 had no significant effect on Huh7 cell proliferation, consistent with previous reports ${ }^{14,22,29}$. Of note, Sec62 knockdown effectively blocked the migration and invasion of Huh7 cells, whereas exogenous expression of Sec62 notably promoted the migration and invasion of Huh7 cells. Similar results were found in $97 \mathrm{H}$ and 97L cells. Consistently, the migration potential of prostate cancer cells, NSCLC cells, thyroid cancer cells, and cervical cancer cells were markedly reduced by Sec62 knockdown, whereas the migration of cervical cancer cells and human embryonic kidney cells were promoted by Sec62 overexpression $^{22,29}$. These effects of Sec62 on cell movement were further confirmed by subsequent $\mathrm{GO}$ analysis with a microarray. These results all indicate a positive link between Sec62 and cell migration, which is essential for surgical recurrence and metastasis of tumours.

Although the above studies indicate a specific relevance of Sec62 to HCC cell migration and invasion, the underlying mechanisms remain unclear. As Sec62 takes part in the protein translocation in the ER, it is reasonable to deduce that a distinct subgroup of migration-relevant precursor proteins rely on Sec62 for efficient transport. However, no substrates have been identified up to now. In this study, the integrin $\alpha /$ Cav1 pathway was identified as a pivotal regulator of Sec62-induced cell migration and 
invasion by microarray analysis. Other Sec62-regulated pathways may also contribute to the recurrence and metastasis of HCC. Integrin $\alpha / C A V 1$ was identified as being down-regulated after Sec62 silencing and upregulated after Sec62 overexpression. Importantly, integrin $\alpha / C A V 1-r e g u l a t e d$ cell movement was the most modulated function following Sec62 knockdown and overexpression according to $\mathrm{GO}$ analysis. Integrins/calpain have been reported to be involved in cell migration and tumour metastasis ${ }^{16,17,22,30-32}$. CAV1 is known to be a general regulator of integrin signalling ${ }^{33}$. qRT-PCR and Western blot analysis confirmed that the expression of integrin $\alpha 2 b$, integrin $\alpha 4$, integrin $\alpha 5$, integrin $\alpha \mathrm{V}$, CAV1, calpain, and MLCK in the integrin $\alpha$ pathway coincided with the expression of Sec62. However, the levels of SHC and SOS in qRT-PCR were not consistent with those in Western blotting. This phenomenon can be explained by (i) the spatial and temporal intervals between the time and loci of transcription and translation of eukaryotic gene expression and (ii) the different half-life of protein than that of mRNA. As a result, integrins, CAV1, calpain, and MLCK might be major regulators of the integrin pathway in this study. We also found that overexpression of upstream genes of integrin signalling, integrin $\alpha 5$ and integrin $\alpha \mathrm{V}$, partially rescued the decreased migration and invasion of HCC cells induced by Sec62 knockdown; however, integrin $\alpha 2$ and integrin $\alpha 4$ did not have a similar function. These results indicate that Sec62promoted cell migration and invasion is dependent on integrin $\alpha / C A V 1$ signalling. However, the correlation between Sec62 and integrin $\alpha$ remains unclear. IP data demonstrated that Sec62 could bind to integrin $\alpha 5$ and integrin $\alpha \mathrm{V}$ and then regulate integrin $\alpha / \mathrm{CAV} 1$ signalling. Importantly, the clinical data strongly suggested that the levels of integrin $\alpha 5$, integrin $\alpha \mathrm{V}$, CAV1, calpain, and MLCK expression, which were positively correlated with Sec62 expression, were higher in HCC early recurrent patients than in non-recurrent patients. Thus, it is reasonable to conclude that integrin $\alpha 5$ and integrin $\alpha \mathrm{V}$ are functional targets of Sec62 and play important roles in Sec62-promoted HCC surgical recurrence and metastasis.

Our in vivo data demonstrated that overexpression of Sec62 promoted postsurgical recurrence and metastasis, whereas Sec62 knockdown significantly decreased HCC recurrence after surgery in an orthotopic HCC xenograft nude mouse model. The expression of Sec62 targets, integrin $\alpha \mathrm{V}$, integrin $\alpha 5$, CAV1, calpains, and MLCK, and Sec62 were in substantial agreement in vivo, which is consistent with those of clinical samples. These findings indicate that Sec62 might be a prognostic biomarker and an attractive therapeutic target for combating HCC postsurgical recurrence.

In conclusion, Sec62 promotes HCC relapse and metastasis by targeting integrin $\alpha / C A V 1$ signalling. Thus, targeting Sec62 in patients who have undergone HCC resection might improve their surgical outcome via effective inhibition of tumour relapse and metastasis.

\section{Materials and methods \\ Cell lines and recombinant virus}

The liver cancer cell lines Huh7, MHCC97-L and MHCC97-H were purchased from Cell Bank of Type Culture Collection of Chinese Academy of Sciences (Shanghai, China). Lentivirus RNA interference of Sec62 (LV-RNAi Sec62 shRNA, 5' - GTTGCTCGATGCATTCT AT $\left.-3^{\prime}\right)$, a negative control lentiviral vector (LV-CON shRNA, 5'-TTCTCCGAACGTGTCACGT-3'), lentiviral packaging plasmids expressing Sec62 (NCBI RefSeq record: NM_003262) and a negative control lentiviral vector were synthesised by GeneChem Co., Ltd. (Shanghai, China). The lentivirus titers were $1 \times 10^{8}$ infectious $\mathrm{U} / \mathrm{ml}$. A rabbit antihuman Sec62 antibody (ab 137022) was procured from Abcam. Rabbit anti-integrin $\alpha 2 b$ (\#13807), integrin $\alpha 4$ (\#8440), integrin $\alpha 5$ (\#98204), integrin $\alpha \mathrm{V}$ (\#60896), CAV1 (\#3267), phospho-SHC (Tyr317, \#2431), phospho-SHC (Tyr239/240, \#2434), SHC (\#2432), SOS1 (\#5890), Bcar3 (\#24032), Calpain 1 (\#2556), Calpain 2 (\#2539), antibodies were procured from Cell Signal Technology. Rabbit antiMLCK (SAB1300116) was procured from Sigma.

\section{Animals}

BALB/c male nude mice ( $20 \pm \mathrm{g}$, 6 weeks) were housed under standard conditions in Zhongshan hospital. Three of four mice were housed in a cage under $12 \mathrm{~h}$ light and $12 \mathrm{~h}$ dark. The animal protocols were performed in agreement with the SIBS Guide for the Care and Use of Laboratory Animals and were approved by the Animal Ethics Committee of Zhongshan Hospital, Fudan University.

\section{Patients}

Tumour samples from $60 \mathrm{HCC}$ patients were obtained from Eastern Hepatobiliary Surgery Hospital during 2016-2017 after written informed consent was obtained. All patients had early HCCs with tumour nodules less than $5 \mathrm{~cm}$ and underwent hepatectomy after their HCC diagnosis. After their discharge from the hospital, all patients were closely followed up. When intrahepatic recurrence was suspected by volumetric computed tomography and/or magnetic resonance imaging, percutaneous liver biopsy with fine-needle aspiration was performed under ultrasound guidance for histological confirmation of the diagnosis. Table 1S summarises the baseline characteristics of the patient cohort. Patients with missing data were excluded from the analysis.

\section{Establishment of a Luciferase-Labelled Orthotopic HCC Model}

Huh7-Sec62 ${ }^{K D}$ or Huh7-Sec62 ${ }^{O E}$ stable transfectants and Huh7-NC cells were established using a lentivirus 
expressing shSec62 or Sec62 and a scramble control with a luciferase reporter gene as described previously ${ }^{34}$. The cells were subcutaneously inoculated into the right flank of nude mice. Three mice per group were for each kind of cells. Three weeks after injection, we isolated subcutaneous tumours and orthotopically grafted them into the livers of nude mice to establish a luciferase-labelled orthotopic HCC model.

The experiments utilised another 30 nude mice, which were randomly allotted to Huh7-Sec62 ${ }^{K D}(n=7)$ and NC $(n=7)$, Huh7-Sec62 $2^{O E}(n=8)$ and NC $(n=8)$ sub groups. A left upper abdominal paramedian incision was made under anaesthesia, the left lobe of the liver was exposed and part of the liver surface was mechanically injured with scissors. A piece of the Huh7 tumour $\sim 1 \mathrm{~mm}$ in diameter was fixed within the liver tissue, and the abdominal wall was closed. On the 14th day after implantation, the lobes where tumours had been implanted were excised from mice. The tumours were $\sim 3 \mathrm{~mm}$ in diameter on the 10th or 14th day. The incisional margin and tumour edge were $\pm 3 \mathrm{~mm}$.

In vivo tumour recurrence was measured with in vivo Imaging System Fx Pro (Carestream Health, USA) according to the location of the luciferase signal during isoflurane anaesthesia in mice. The investigator was blinded to the group allocation during the image exposure period. As anticipated, signals emanating from the liver area indicated intrahepatic recurrence, whereas signals outside the liver area indicated abdominal metastasis.

\section{Wound-healing assay}

Huh7-Sec6 $2^{K D}$ or Huh7-Sec62 ${ }^{O E}$ cells were implanted into 6-well culture dishes. When the cells grew to $90 \%$ confluence, a sterilised tip was used to draw a line with the same width on the bottom of the dishes. Images were captured at $0,24,48 \mathrm{~h}$ after wounding. The data shown are representative of three independent repeats.

\section{Migration and invasion assays}

Transwell cell migration was quantified by seeding cells $\left(n=5 \times 10^{4}\right)$ in serum-free medium containing $0.1 \%$ bovine serum albumin onto the top layer of a 24-well BD BioCoat Matrigel invasion chamber (BD Biosciences) for quantitative assessment of cell invasion. After $24 \mathrm{~h}$, cells were stained and counted on the lower side of the membrane under a light microscope $(\times 200,10$ random fields from each well). All experiments were conducted in quadruplicate.

\section{Immunohistochemical staining}

Immunohistochemical (IHC) was performed on formalin-fixed, paraffin-embedded human tissue sections as previously described ${ }^{35,36}$. Sections were incubated with an anti-Sec62 antibody in a humidified chamber at $4{ }^{\circ} \mathrm{C}$ overnight. The following staining was performed using the Envision plus System according to the manufacturer's instructions. Tissue sections were counterstained with hematoxylin and analysed using light microscopy (Nikon). The staining scores of Sec62 were evaluated by Image-Pro Plus 6.0 based on the integral optical density (IOD). The staining intensity was graded as follows: 1 , no staining $(\mathrm{IOD}=0)$; 2 , weak staining $(\mathrm{IOD}<150)$; 3 , moderate staining $\quad(150 \leq \mathrm{IOD}<1000) ;$ and 4 , strong staining $(\mathrm{IOD} \geq 1000)$. A score $\leq 2$ was defined as low expression of Sec62, and a score $>2$ was defined as high expression of Sec62. The outcome assessing was done by another researcher who did not know the follow-up results.

\section{Co-immunoprecipitation of Sec62}

Huh7 cells were lysed in lysis buffer containing $50 \mathrm{mM}$ HEPES, $150 \mathrm{mM} \mathrm{NaCl}$ (4.38 g), $1 \mathrm{mM}$ EDTA, 1\% (w/v) CHAPS and Sigma protease inhibitor cocktail (with or without $50 \mu \mathrm{g} / \mathrm{ml}$ of RNaseA) at room temperature for $10 \mathrm{~min}$. M2 FLAG agarose resin $(40 \mu \mathrm{l})$ was prepared as described by the manufacturer's instructions and incubated with $1000 \mu \mathrm{l}$ of the cell lysate supernatant with gentle agitation at $4{ }^{\circ} \mathrm{C}$ overnight. The IP samples were spun down at $8000 \mathrm{rpm}$ for $30 \mathrm{~s}$ and washed with wash buffer (50 mM HEPES, $150 \mathrm{mM} \mathrm{NaCl}, 0.1 \%$ Triton X-100, $10 \%$ glycerol, $\mathrm{pH}$ to 7.5$)$ three times. Then, the proteins were eluted with FLAG peptide $(200 \mathrm{ng} / \mu \mathrm{l})$ and transferred into a new tube for further analysis.

\section{Statistical analyses}

Statistical analyses were performed using SPSS version 17.0 (SPSS, Chicago, IL). All data were presented as the means \pm standard deviation (S.D). The $\chi^{2}$ test, Fisher's exact probability test, and Student's $t$ test were used for comparisons between groups. The probability of recurrence-free survival was analysed by the Kaplan-Meier method, and differences between groups were estimated by the log-rank test. $p<0.05$ (two-tailed) was considered statistically significant. The predictive accuracy was calculated using the ROC. For animal studies, sample size was estimated to be at least seven mice per group to ensure power with statistical confidence Statistical differences between two groups.

\section{Acknowledgements}

We would like to thank Yuan Wang for technical assistance and Feng Yang of Huashan Hospital and Mack Y. Su of Massachusetts General Hospital, Harvard Medical School for their assistance with the preparation of the paper. This work was supported by the National Nature Science Foundation of China (grant number: 81473484 and 81430101 ) and the Nature Science Foundation of Shanghai (grant number: 14ZR1408400).

\section{Author details}

${ }^{1}$ Department of Traditional Chinese Medicine, Changhai Hospital, Navy Military Medical University, Shanghai 200433, China. ${ }^{2}$ The Third Department of Hepatic Surgery, Eastern Hepatobiliary Surgery Hospital, Navy Military Medical University, Shanghai 200433, China. ${ }^{3}$ Liver Cancer Institute, Zhongshan Hospital, Fudan University, Shanghai 200433, China 


\section{Authors' contributions}

Concept and design: C.Q.L. and J.D. Data collection: J.D., Z.Z.H., D.L., H.T.Z., B.B. C., J.C., H.L. and X.F.Z. Statistical analysis: J.D., D.L., Z.Z.H. and H.T.Z. Writing of article: all authors.

\section{Conflict of interest}

The authors declare that they have no conflict of interest.

\section{Ehtical approval}

The study was approved by Changhai Hosipital Ethic Committee in Changhai Hospital, Eastern Hepatobiliary Surgery Hospital, Navy Military Medical University and Zhongshan Hospital, Fudan University. The study had been registered with Chinese Clinical Trials Registry, number ChiCTR-TRC-07000033 and was performed in accordance with the Declaration of Helsinki. Animal experiments were approved by Animal Ethics Committee of Zhongshan Hospital. Only male mice were used since the study aimed to identify mechanisms and a prognostic biomarker of HCC recurrence, which is high in male.

\section{Publisher's note}

Springer Nature remains neutral with regard to jurisdictional claims in published maps and institutional affiliations.

Supplementary Information accompanies this paper at (https://doi.org/ 10.1038/s41389-019-0183-6).

Received: 15 July 2019 Revised: 19 November 2019 Accepted: 25 November 2019

Published online: 10 December 2019

\section{References}

1. Bray F. et al. Global cancer statistics 2018: GLOBOCAN estimates of incidence and mortality worldwide for 36 cancers in 185 countries. CA: a cancer journal for clinicians (2018).

2. Chimed, T. et al. Cancer incidence and cancer control in Mongolia: results from the National Cancer Registry 2008-12. Int. J. Cancer 140, 302-309 (2017).

3. Bertuccio, P. et al. Global trends and predictions in hepatocellular carcinoma mortality. J. Hepatol. 67, 302-309 (2017).

4. Ferlay, J. et al. Cancer incidence and mortality worldwide: sources, methods and major patterns in GLOBOCAN 2012. Int. J. Cancer 136, E359-E386 (2015).

5. Chan, A. C. et al. Treatment strategy for recurrent hepatocellular carcinoma: salvage transplantation, repeated resection, or radiofrequency ablation? Liver Transpl. 19, 411-419 (2013).

6. Toyoda, H. et al. Long-term impact of liver function on curative therapy for hepatocellular carcinoma: application of the ALBI grade. Br. J. Cancer 114, 744-750 (2016).

7. Lim C. et al. Impact of time to surgery in the outcome of patients with liver resection for BCLC 0-A stage hepatocellular carcinoma. J. Hepatol. pii: S0168-8278(17)32331-0 (2017). [Epub ahead of print].

8. Ling, C. Q. et al. Clinical practice guidelines for the treatment of primary liver cancer with integrative traditional Chinese and Western medicine. J. Integr. Med. 16, 236-248 (2018).

9. Nishida N. et al. Molecular scoring of hepatocellular carcinoma for predicting metastatic recurrence and requirements of systemic chemotherapy. Cancers 10, 367 (2018).

10. Olugbami, J. O. et al. Atomic force microscopy correlates antimetastatic potentials of HepG2 cell line with its redox/energy status: effects of curcumin and Khaya senegalensis. J. Integr. Med. 15, 214-230 (2017).

11. Tyedmers, J. et al. Homologs of the yeast Sec complex subunits Sec62p and Sec63p are abundant proteins in dog pancreas microsomes. Proc. Natl Acad. Sci. USA 97, 7214-7219 (2000).

12. Dudek, J. et al. ERj1p has a basic role in protein biogenesis at the endoplasmic reticulum. Nat. Struct. Mol. Biol. 12, 1008-1014 (2005).

13. Muller, L. et al. Evolutionary gain of function for the ER membrane protein Sec62 from yeast to humans. Mol. Biol. Cell. 21, 691-703 (2010).
14. Greiner, M. et al. Silencing of the SEC62 gene inhibits migratory and invasive potential of various tumor cells. Int. J. Cancer 128, 2284-2295 (2011).

15. Li, H. et al. Hypoxia-inducible factor 1 alpha-activated angiopoietin-like protein 4 contributes to tumor metastasis via vascular cell adhesion molecule-1/ integrin beta1 signaling in human hepatocellular carcinoma. Hepatology $\mathbf{5 4}$ 910-919 (2011).

16. Cai, Q. et al. SIN3B promotes integrin alphaV subunit gene transcription and cell migration of hepatocellular carcinoma. J. Mol. Cell Biol. 11, 421-432 (2018).

17. $W u, W$. et al. Regulation of integrin alphaV subunit expression by sulfatide in hepatocellular carcinoma cells. J. Lipid Res. 54, 936-952 (2013).

18. Ma, J. et al. Human U87 astrocytoma cell invasion induced by interaction of betaig-h3 with integrin alpha5beta1 involves calpain-2. PLOS ONE 7, e37297 (2012).

19. Zeltz C. et al. a $11 \beta 1$ integrin is induced in a subset of cancer-associated fibroblasts in desmoplastic tumor stroma and mediates in vitro cell migration. Cancers 11, E765 (2019).

20. Zhang, X. B. et al. Upregulation of microRNA-31 targeting integrin alpha5 suppresses tumor cell invasion and metastasis by indirectly regulating PIJKJAKT pathway in human gastric cancer SGC7901 cells. Tumour Biol. 37, 8317-8325 (2016)

21. Chen, B. et al. Calpains are required for invasive and metastatic potentials of human HCC cells. Cell. Biol. Int. 37, 643-652 (2013).

22. Linxweiler, $M$. et al. Sec62 bridges the gap from $3 q$ amplification to molecular cell biology in non-small cell lung cancer. Am. J. Pathol. 180, 473-483 (2012)

23. Kim, D. Y. \& Helfman, D. M. Loss of MLCK leads to disruption of cell-cell adhesion and invasive behavior of breast epithelial cells via increased expression of EGFR and ERK/JNK signaling. Oncogene 35, 4495-4508 (2016).

24. Chan, A. W. et al. Prognostic Nutritional Index (PNI) predicts tumor recurrence of very early/early stage hepatocellular carcinoma after surgical resection. Ann. Surg. Oncol. 22, 4138-4148 (2015).

25. Linxweiler, M. et al. Targeting cell migration and the endoplasmic reticulum stress response with calmodulin antagonists: a clinically tested small molecule phenocopy of SEC62 gene silencing in human tumor cells. BMC Cancer 13, 574 (2013).

26. Wemmert, S. et al. Initial evidence for Sec62 as a prognostic marker in advanced head and neck squamous cell carcinoma. Oncol. Lett. 11, 1661-1670 (2016).

27. Greiner, M. et al. Sec62 protein level is crucial for the ER stress tolerance of prostate cancer. Prostate 71, 1074-1083 (2011).

28. Jung, V. et al. Genomic and expression analysis of the 3q25-q26 amplification unit reveals TLOC1/SEC62 as a probable target gene in prostate cancer. Mol. Cancer Res. 4, 169-176 (2006).

29. Linxweiler, M. et al. Identification of SEC62 as a potential marker for 3q amplification and cellular migration in dysplastic cenvical lesions. BMC Cancer 16,676 (2016).

30. Bhandari, A. et al. ITGA7 functions as a tumor suppressor and regulates migration and invasion in breast cancer. Cancer Manag. Res. 10, 969-976 (2018).

31. McHugh, B. J., Murdoch, A., Haslett, C. \& Sethi, T. Loss of the integrin-activating transmembrane protein Fam38A (Piezo1) promotes a switch to a reduced integrin-dependent mode of cell migration. PLOS ONE 7, e40346 (2012).

32. Almeida, M. E. et al. Hyperglycemia reduces integrin subunits alpha $v$ and alpha 5 on the surface of dermal fibroblasts contributing to deficient migration. Mol. Cell. Biochem. 421, 19-28 (2016).

33. Chapman, H. A., Wei, Y., Simon, D. I. \& Waltz, D. A. Role of urokinase receptor and caveolin in regulation of integrin signaling. Thromb. Haemost. 82, 291-297 (1999).

34. Huang, W. et al. Sox12, a direct target of FoxQ1, promotes hepatocellular carcinoma metastasis through up-regulating Twist1 and FGFBP1. Hepatology 61, 1920-1933 (2015)

35. Cui, Y. M. et al. FOXC2 promotes colorectal cancer metastasis by directly targeting MET. Oncogene 34, 4379-4390 (2015).

36. Bao, X. X. et al. Preliminary optimization of a Chinese herbal medicine formula based on the neuroprotective effects in a rat model of rotenone-induced Parkinson's disease. J. Integr. Med. 16, 290-296 (2018). 